\title{
An unusual cause of cervical dysphagia
}

de Silva W.D.D, $\mathrm{MBBS}^{1}$, Kumara M.T.G.J, MBBS, $\mathrm{MD}^{2}$, de Silva W.M.M, MBBS, MS, FRCS. ${ }^{3}$

1 Surgical Registrar, University Surgical Unit, Colombo South Teaching Hospital.

2 Senior Surgical Registrar, University Surgical Unit, Colombo South Teaching Hospital.

3 Professor and Head, Department of Surgery, University of Sri Jayewardenepura.

Key words: Dysphagia; Osteophyte.

\section{Introduction}

Dysphagia, presenting in the elderly arouses suspicion of a malignant lesion in the oesophagus which is the usual cause. Occasionally, a benign lesion such as a hiatus hernia may also be responsible. We report a case of dysphagia which proved to be from a more unusual cause.

\section{Case report}

An 82 year old male presented with a history of dysphagia for solids of six months duration. He had had a good appetite and examination revealed a thin but relatively healthy man with normal neck movements. Neurological examination was normal.

An upper gastro-intestinal endoscopy was performed. A bulge was noticed about $4 \mathrm{~cm}$ below the cricopharyngeal junction but no internal lesion was detected. Histology of the oesophageal mucosa over the bulge proved unremarkable. A Barium swallow revealed a narrowing of the oesophagus at $\mathrm{C} 5 / \mathrm{C} 6$ level caused by impingement from a prominent anterior cervical osteophyte (Figure 1). No other lesion was found. The patient did not want surgical intervention raising a dilemma about appropriate management.

Correspondnce: W.D.D. de Silva, Registrar, University Surgical Unit, Colombo South Teaching Hospital.

Email: desilva.dulantha@gmail.com

The Sri Lanka Journal of Surgery 2010; 28(2):25-26.

\section{Discussion}

Dysphagia due to anterior cervical osteophytes is rare. The oesophagus is relatively fixed at the level of the cricoid cartilage and at the level of the diaphragm, while the remainder of the oesophagus is mobile. Thus, bony vertebral projections impinging at the level of the cricopharyngeus may cause dysphagia due to the relative immobility of this point [1].

Peri-oesophageal oedema and local inflammatory reaction, causing spasm, may also contribute to the dysphagia. Occasionally such osteophytes may even cause dyspnoea,

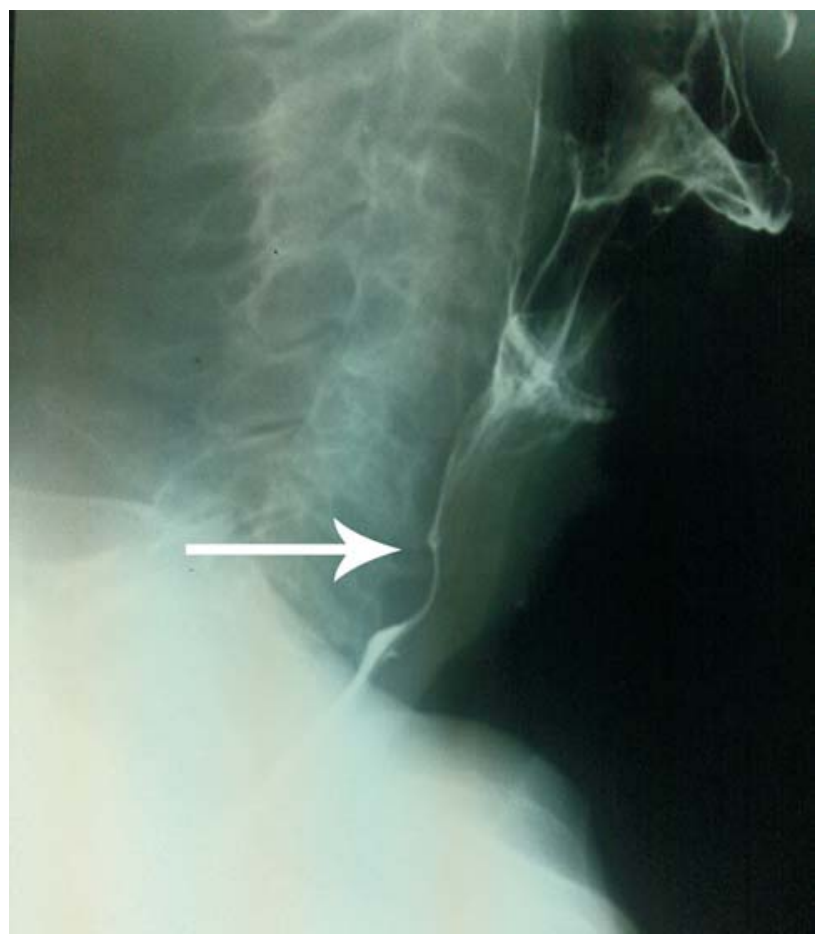

Figure 1. Barium Swallow showing a prominent osteophyte at C5/C6 level impinging on the cervical oesophagus (White arrow) 
pharyngeal fistulae or obstructive sleep apnoea. [2]

The causes of hypertrophic anterior cervical osteophytes include cervical spondylosis, diffuse hypertrophic spinal hyperostosis (DISH), ankylosing spondylitis, inter-vertebral disc degeneration, trauma and infection [3].

Cervical osteophytes are present in up to 20$30 \%$ of the elderly population but do not usually cause symptoms. Although dysphagia has been reported in the literature, many have been associated with DISH [4]. Interestingly, in our patient, the clinical profile was more in keeping with spondylosis rather than DISH.

Diagnosis is facilitated by lateral cervical $\mathrm{x}$ rays, Barium swallow and CT scans. Oesophagoscopy is required to exclude intrinsic lesions but should be performed with care in view of the risk of perforation. It is always important to exclude more sinister causes, like cancer, before attributing the cause of dysphagia to osteophytes [3].

Management of dysphagia from cervical osteophytes depends on the degree of symptoms. Milder dysphagia may be managed conservatively with non-steroidal antiinflammatory drugs (NSAIDS), dietary modification and observation [3]. In patients unable to tolerate oral NSAIDS, because of swallowing difficulty, phonophoresis involving transdermal NSAID delivery enhanced by ultrasound appears effective [5]. In severe cases however, surgical excision of the osteophyte, usually via an anterior cervical approach, has been associated with very good results $[2,6]$.

\section{References}

1. Lambert JR, Tepperman PS, Jimenez J, Newman A. Cervical spine disease and dysphagia: Four new cases and a review of the literature. American Journal of Gastroenterology 1981; 76:35-40.

2. Fuerderer S, Eysel-Gosepath K, Schröder U, Delank KS, Eysel P. Retro-pharyngeal obstruction in association with osteophytes of the cervical spine. Journal of Bone and Joint Surgery (Br.) 2004; 86:837-40.

3. Srinivas P, George J. Cervical osteoarthropathy: an unusual cause of dysphagia. Age and Ageing.1999; 28:3212.

4. Giger R, Dulguerov P, Payer M. Anterior cervical osteophytes causing dysphagia and dyspnea: an uncommon entity revisited. Dysphagia 2006; 21:259-63.

5. Unlu Z, Orguc S, Eskiizmir G, Aslan A, Tasci S.The role of phonophoresis in dysphagia due to cervical osteophytes. International Journal of General Medicine 2009; 1:11-3.

6. Oppenlander ME, Orringer DA, La Marca F et al. Dysphagia due to anterior cervical hyperosteophytosis. Surgical Neurology 2009; 72:266-70.

\section{Key Learning Points}

The oesophagus is fixed at the level of the cricopharynx and at the diaphragm.

Barium swallow may complement endoscopic examination in evaluation of dysphagia. 